\title{
Satellite Image Resolution Enhancement Using Double Density Dual-Tree Complex Wavelet Transform and Nonlocal Means
}

\author{
Ritesh. S. Patil ${ }^{1}$, Mr. Pravin. S. Patil ${ }^{2}$ \\ P.G. Student SSVP's College of Engineering and Research, Dhule (M.H), India ${ }^{1}$ \\ SSVP's College of Engineering and Research, Dhule (M.H), India ${ }^{2}$
}

\begin{abstract}
A Multi scale decomposition based on double density dual-tree complex wavelet transform and edge preservation is presented for SRE of the satellite images. Resolution enhancement schemes (which are not based on wavelets) suffer from the drawback of losing high frequency contents (which results in blurring). The Discrete Wavelet Transform- based (DWT) RE scheme generates artifacts (due to a DWT shift-variant property). A wavelet-domain approach based on Double Density Dual-Tree Complex Wavelet Transform (DDDTCWT) and nonlocal means is used for RE of the satellite images. A satellite input image is decomposed by DDDTCWT to obtain high-frequency subbands. The high-frequency subband and the low-Resolution (LR) input image are interpolated using the bicubic interpolator. The high frequency subbands are passed through an NLM filter to cater for the artifacts generated by DDDTCWT. The filtered high-frequency subbands and the LR input image are combined using inverse DDDTCWT to obtain a resolution-enhanced image. The simulated results will show that technique used in this process provides better accuracy rather than prior methods.
\end{abstract}

Keywords: Pre-processing, double density dual-tree complex wavelet transform (DDDTCWT) nonlocal means filter (NLM), Reconstruction, Parameter analysis.

\section{I.INTRODUCTION}

Image enhancement problem can be formulated as follows: given an input low quality video and the output high quality video for specific applications. How can we make video more clearer or subjectively better? Digital video has become an integral part of everyday life. As the use of large displays is increasing, the demand for higher quality videos is growing fast in consumer market. New devices have been implemented to capture images and videos with much finer details. To this end more powerful optics and complex image stabilization mechanisms are required. Although many improvements have been made over the capturing devices and cameras, further enhancements are subjected to hardware complexity and restrictions.

In many applications including cell phone and webcam the imaging sensors capture low resolution images due to low cost sensors or physical limitation of the hardware and then a software alternative improves the quality of the captured frames. There are techniques to increase the resolution in an offline manner after that the image or video has been captured. Super-resolution (SR) is among techniques to improve the quality of the images received by the users of consumer applications such as video streaming on the Internet, cell phone devices and video conferencing. Super Resolution makes a high resolution frame out of one or a set of Low Resolution (LR) frames. In fact in low resolution images the high frequency components are missed and SR tries to estimate those missing frequencies in a way that the difference between the original image and the reconstructed SR is minimum [1].

There are different methods to generate HR images from the LR ones. Some methods used the reconstruction based approaches and some others use the learning algorithms. Reconstruction based approaches use the information in separate frames for solving the problem of SR. In fact these methods use the independent information in different frames and fuse them to make one HR image. Projection onto Convex Sets (POCS) [2] and Iterative Back-Projection [3] Methods are among the famous SR+ methods in literature. Learning based approaches use a specific image sequence set to learn their characteristics and use those known characteristics as extra information to reconstruct the HR image. Recently there are some new methods introduced which only use a single LR image for finding the HR image. 


\section{International Journal of Innovative Research in Electrical, Electronics, Instrumentation and Control Engineering}

Vol. 6, Issue 12, December 2018

\section{LITERATURE SURVEY}

\section{A. Discrete Wavelet Transform (DWT)}

Discrete Wavelet Transform is any wavelet transform which uses wavelet coefficients. The DWT technique which captures both frequency and location information of an image. Resolution is an important feature in satellite imaging. The satellite image have high frequency contents as well as low frequency contents. And the image may have losing of high frequency contents. So, the DWT technique has been employed for resolution to preserve the high frequency components of the satellite images [10]. The process is to divide the satellite input image into four sub bands. They are Low-Low(LL), Low-High(LH), High- Low (HL), High-High (HH). Then the high frequency sub bands are estimated. The high frequency sub band images and the low resolution input images are interpolated and using inverse DWT we can get a resolution enhanced image [4]. The interpolation process is used to preserve high frequency contents of the image. The DWT technique is mainly used to produce the sharper enhanced image.

\section{B. Stationary Wavelet Transform (SWT)}

The Stationary Wavelet Transform is used to overcome the lack of translation-variance of the Discrete Wavelet Transform (DWT). And SWT technique is redundant. As like DWT, the SWT also divides the input image into different sub bands. The high frequency sub bands obtained by DWT and SWT are added with each other which mean they have the same size [13]. That is, the interpolated high frequency sub band coefficients have been corrected by the SWT high frequency sub band coefficients [5]. After that, the images are combined using inverse process to get resolution enhanced image. So, the technique generates a super resolved image.

\section{Inter Sub band Correlation Technique (ISC)}

This method which uses same phase for the sub bands. So, the sampling phase is considered. The method has the filter bank to estimate the sub bands [11]. The sub bands have correlation that is between low frequency band and high frequency band. If we have different phases, the sub bands will have low correlation with one another. This method has three steps. They are,

1) First apply the wavelet transform to all different phases of each sub band.

2) The filters are used to estimate the bands in higher level.

3) Inverse wavelet transform is applied to enhance the resolution of an input image.

Thus, using the same phase for estimating the sub bands, this method will produce a time consuming process.

\section{DCT}

The DCT transforms or converts a signal from spatial domain into a frequency domain. DCT is real-valued and provides a better approximation of a signal with few coefficients. This approach reduces the size of the normal equations by discarding higher frequency DCT coefficients. Important structural information is present in the low frequency DCT coefficients. Hence, separating the high-frequency DCT coefficient and applying the illumination enhancement in the low-frequency DCT coefficient, it will collect and cover the edge information from satellite images. The enhanced image is reconstructed by using inverse DCT and it will be sharper with good contrast.

\section{PROPOSED METHOD}

The proposed system has mainly four modules: Pre-processing, Double Density Dual-Tree Complex Wavelet Transform (DDDTCWT) nonlocal means filter (NLM) and Interpolation.

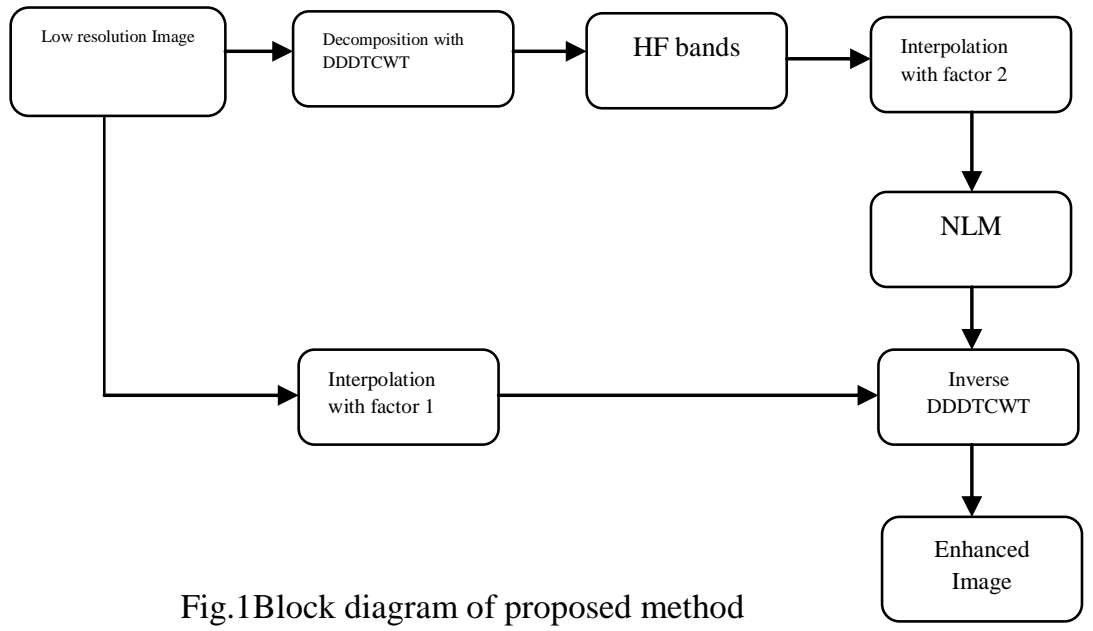




\title{
International Journal of Innovative Research in Electrical, Electronics, Instrumentation and Control Engineering
}

Vol. 6, Issue 12, December 2018

\author{
Algorithm: \\ 1. Apply Double Density Dual Tree CWT multi scale decomposition for Input image. \\ 2. Separate Low and High Freq components. \\ 3. Apply Lanczos interpolation with beta/2 for input image. \\ 4. Apply Lanczos interpolation with beta and Nlm filter for High freq comp. \\ 5. Reconstruct Low and High Freq components. \\ 6. Apply Inverse Double Density Dual Tree CWT. \\ 7. Validate MSE and PSNR.
}

\section{Double-Density Complex Wavelet Transform}

Both the double-density DWT and the dual-tree DWT have their own distinct characteristics and advantages, and as such, it was only natural to combine the two into one transform called the double-density complex (or double-density dual-tree) DWT. To combine the properties of both the double-density and dual-tree DWTs we ensure that: (1) one pair of the four wavelets is designed to be offset from the other pair of wavelets so that the integer translates of one wavelet pair fall midway between the integer translates of the other pair (Eq. 1), and (2) one wavelet pair is designed to be approximate Hilbert transforms of the other pair of wavelets (as stated in [2]). By doing this, we are then able to use the double-density complex wavelet transform to implement complex and directional wavelet transforms.

To implement the double-density dual-tree DWT, we must first design an appropriate filter bank structure (one that combines the characteristics of the double-density and dual-tree DWTs). We have seen what type of filter bank structure is associated with the double-density DWT in the previous sections (mainly that it is composed of one low pass scaling filter and two high pass wavelet filters), so we will now turn to the properties of the dual-tree DWT. The dual-tree DWT is based primarily on concatenating two critically sampled DWTs. We do this by constructing a filter bank that performs multiple iterations in parallel. More detail about the dual-tree DWT can be found at this website.

Consequently, the filter bank structure corresponding to the double-density complex DWT consists of two oversampled iterated filter banks operating in parallel on the same input data. (The oversampled filter bank is illustrated in Fig. 1 in this section.) The iterated oversampled filter bank pair, corresponding to the simultaneous implementation of the double-density and dual-tree DWTs, is illustrated in Figure 2 below.

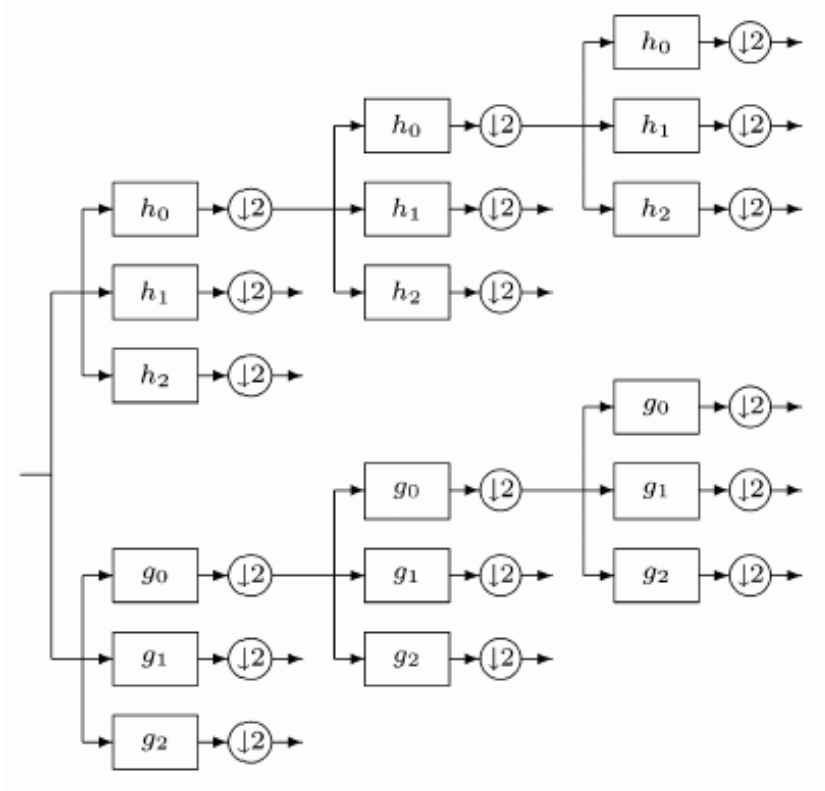

Figure 2. Iterated Filterbank for the Double-Density Complex DWT.

As you can see, there are two separate filter banks denoted by hi(n) and gi(n) where $\mathrm{i}=0,1,2$. The filter banks hi(n) and gi(n) are unique and designed in a specific way so the subband signals of the upper DWT can be interpreted as the real part of a complex wavelet transform, and the subband signals of the lower DWT can be interpreted as the imaginary part. Equivalently, for specially designed sets of filters, the wavelets associated with the upper DWT can be 


\section{IJIREEICE}

\section{International Journal of Innovative Research in Electrical, Electronics, Instrumentation and Control Engineering}

Vol. 6, Issue 12, December 2018

approximate Hilbert transforms of the wavelets associated with the lower DWT. When designed in this way, the double-density complex DWT can be used to implement 2-D oriented wavelet transforms, which are especially efficient in image processing. (Recall that for the double-density DWT, four out of the eight wavelets did not have a dominant orientation.) Because of this, the double-density complex DWT is expected to outperform the double-density DWT in various applications, such as image denoising and enhancement. But first we must design the filters for the double-density complex DWT.

The FIR perfect reconstruction filter banks designed for this procedure are displayed below. We implement them by using one set of filter banks for the first stage (FSdoubledualfilt.m) and a second set of filters for the remaining stages (doubledualfilt.m). The filter bank for the remaining stages is designed such that the analysis filters of the first tree are the synthesis filters for the second tree, and vice versa. The function FSdoubledualfilt.m provides analysis and synthesis filter for the first stage of the double-density oriented DWT, while the function doubledualfilt.m provides analysis and synthesis filters for the remaining stages of the transform.

\section{Non Local Means Approach:}

Non-local means filter is an algorithm in image processing for image denoising. Unlike other local smoothing filters, non-local means filter averages all observed pixels to recover a single pixel. The weight of each pixel depends on the distance between its intensity grey level vector and that of the target pixelThe NLM filter is based on the assumption that image content islikely to repeat itself within some neighbourhood (in the image)and in neighbouring frames. It computes denoisedpixel $\mathrm{x}(\mathrm{p}, \mathrm{q})$ by the weighted sum of the surrounding pixels of $\mathrm{Y}(\mathrm{p}, \mathrm{q})$ (within frame and in the neighbouring frames). Thisfeature provides a way to estimate the pixel value from noisecontaminatedimages. In a 3-D NLM algorithm, the estimate ofa pixel at position $(p, q)$ is

$$
x(p, q)=\frac{\sum_{m=1}^{M} \sum_{(r, s) \in N(p, q)} Y_{m}(r, s) K_{m}(r, s)}{\sum_{m=1}^{M} \sum_{(r, s) \in N(p, q)} K_{m}(r, s)}
$$

Wheremis the frame index, and $\mathrm{N}$ represents the neighbourhood of the pixel at location (p, q). $\mathrm{K}$ values are the filter weights,i.e,

$$
\begin{aligned}
& K(r, s)=\exp \left\{-\frac{\|V(p, q)-V(r, s)\|_{2}^{2}}{2 \sigma^{2}}\right\} \\
& \times f\left(\sqrt{(p-r)^{2}+(q-s)^{2}+(m-1)^{2}}\right)
\end{aligned}
$$

WhereV is the window [usually a square window centred atthe pixels $\mathrm{Y}(\mathrm{p}, \mathrm{q})$ and $\mathrm{Y}(\mathrm{r}, \mathrm{s})$ ] of pixel values from a geometricneighbourhood of pixels $\mathrm{Y}(\mathrm{p}, \mathrm{q})$ and $\mathrm{Y}(\mathrm{r}, \mathrm{s}), \sigma$ is the filter coefficient, and $\mathrm{f}($.$) is a geometric distance$ function. $\mathrm{K}$ isinversely proportional to the distance between $\mathrm{Y}(\mathrm{p}, \mathrm{q})$ and $\mathrm{Y}(\mathrm{r}, \mathrm{s})$. where $\mathrm{Z}$ is the blurred version of the targeted image, and On is the weight matrix, followed by minimizing a deblurringe quation In the proposed algorithm (DT-CWT-NLM-RE), we decomposethe LR input image (for the multichannel case, eachchannel is separately treated) in different subbands (i.e., Ciand $\mathrm{Wji}$, where $\mathrm{i} \in\{\mathrm{A}, \mathrm{B}, \mathrm{C}, \mathrm{D}\}$ and $\mathrm{j} \in\{1,2,3\}$ ) by usingDT-CWT, Civalues are the image coefficientsubbands, and $\mathrm{Wji}$ are the wavelet coefficient subbands.The subscripts $\mathrm{A}, \mathrm{B}, \mathrm{C}$, and D represent the coefficients atthe even-row and even-column index, the odd-row and evencolumnindex, the even-row and odd-column index and theodd-row and odd-column index, respectively, whereas $h$ andg represent the low-pass and high-pass filters, respectively. The superscript e and o represent the even and odd indices,respectively.Wji values are interpolated by factor $\beta$ using the Lanczosinterpolation (having good approximation capabilities) andcombined with the $\beta / 2$ interpolated LR input image. SinceCicontains low-pass-filtered image of the LR input image,therefore, high-frequency information is lost. To cater for it, wehave used the LR input image instead of Ci.Although the DT-CWT is almost shift invariant, however,it may produce artifacts after the interpolation of $\mathrm{Wji}$.Therefore, to cater for these artifacts, NLM filtering is used.All interpolated Wji values are passed through the NLM filter.Then, we apply the inverse DT-CWT to these filtered subbandsalong with the interpolated LR input image to reconstructthe HR image. The results presented show that the proposedDT-CWT-NLM-RE algorithm performs better than the existingwavelet-domain RE algorithms in terms of the peak-signal-tonoiseratio (PSNR), the MSE, and the Q-index.

\section{Interpolation:}

Interpolation is the process of estimating the values of a continuous function from discrete samples. Image processing applications of interpolation include image magnification or reduction, subpixel image registration, to correct spatial distortions, and image decompression, as well as others. Of the many image interpolation techniques available, nearest 


\title{
International Journal of Innovative Research in Electrical, Electronics, Instrumentation and Control Engineering
}

\author{
Vol. 6, Issue 12, December 2018
}

neighbour, bilinear and cubic convolution are the most common, and will be talked about here. Sinc Interpolation provides a perfect reconstruction of a continuous function, provided that the data was obtained by uniform sampling at or above the Nyquist rate. Sinc Interpolation does not give good results within an image processing environment, since image data is generally acquired at a much lower sampling rate. The mapping between the unknown high-resolution image and the low-resolution image is not invertible, and thus a unique solution to the inverse problem cannot be computed. One of the essential aspects of interpolation is efficiency since the amount of data associated with digital images is large.

\section{Parameter Analysis:}

We calculate the value for Root Mean Square Error (RMSE), Peak Signal to Noise Ratio (PSNR) for Enhanced image. using below equations,

$$
P S N R=10 \log _{10}\left(\frac{\max ^{2}}{M S E}\right)
$$

Where,

$$
M S E=\frac{1}{m n} \sum_{i=0}^{m-1} \sum_{j=0}^{n-1}[I(i, j)-K(i, j)]^{2}
$$

\section{RESULT'S}
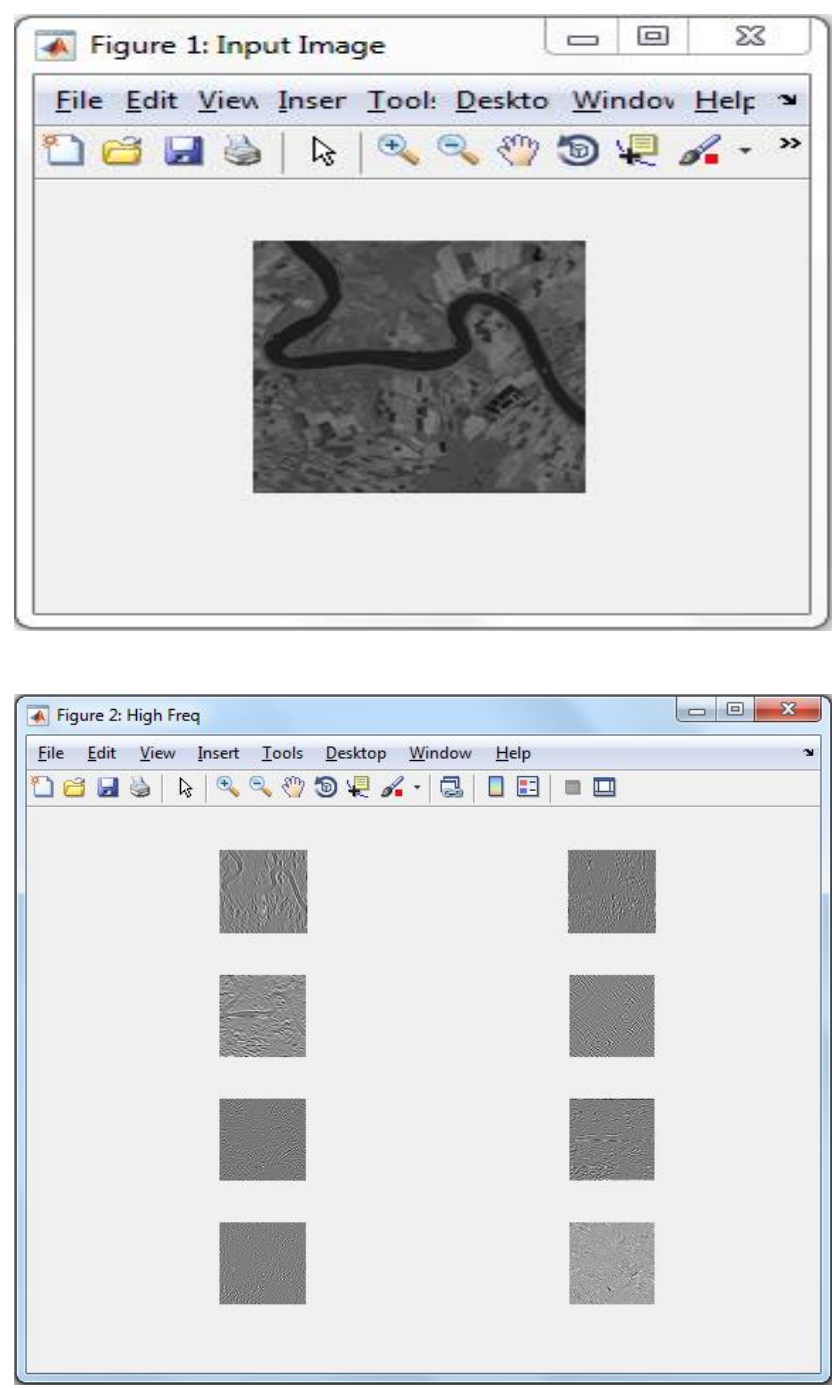
International Journal of Innovative Research in Electrical, Electronics, Instrumentation and Control Engineering

Vol. 6, Issue 12, December 2018
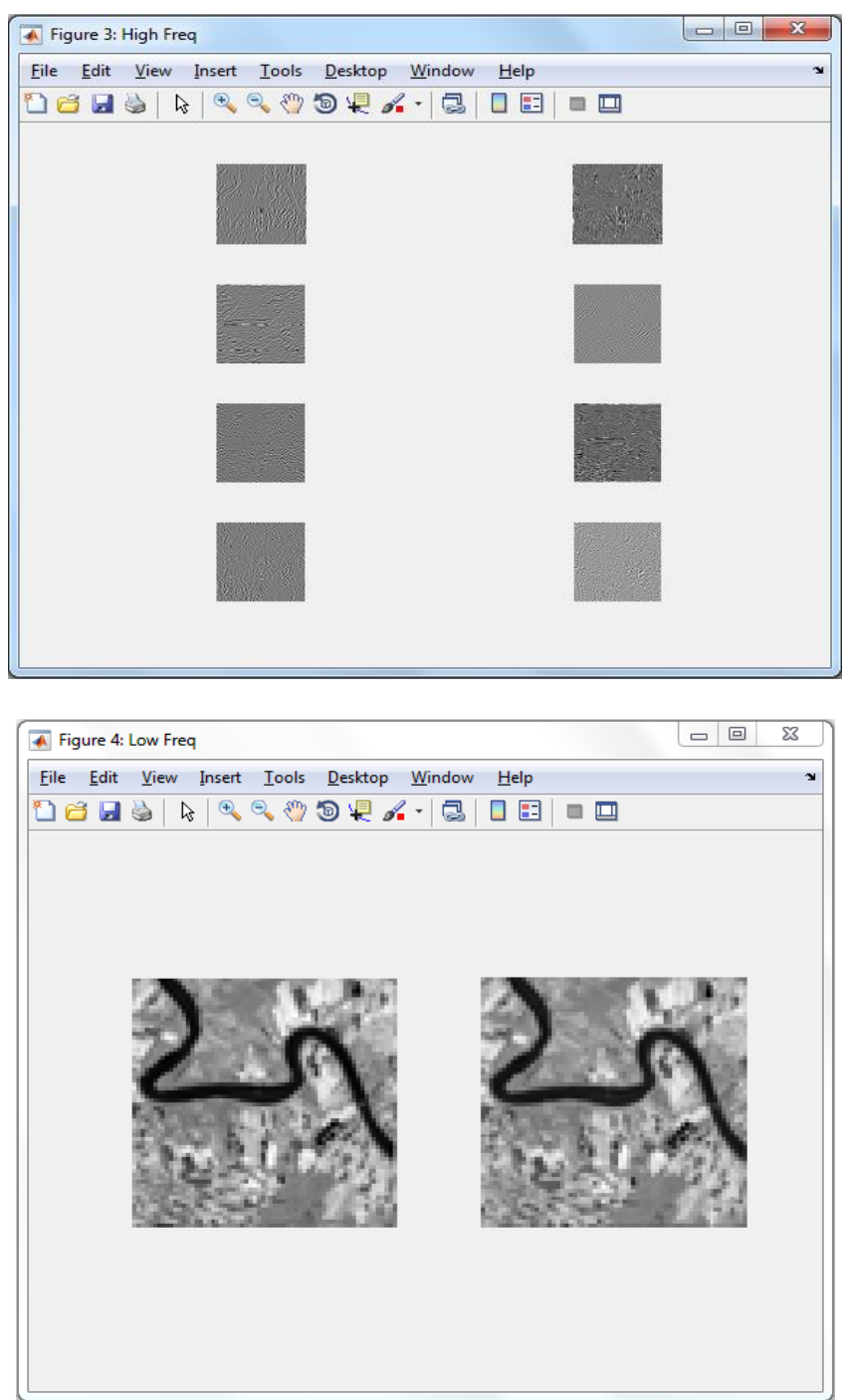

Figure 5: Enhanced Image

\begin{tabular}{|l|l|l|}
\hline$口$ & 回 & $\mathrm{x}$ \\
\hline
\end{tabular}

File Edit Vien Inser Iool: Deskto Windor HelF $\mathbf{v}$

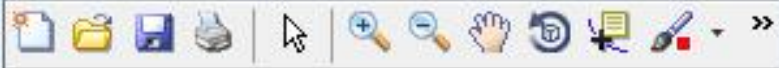

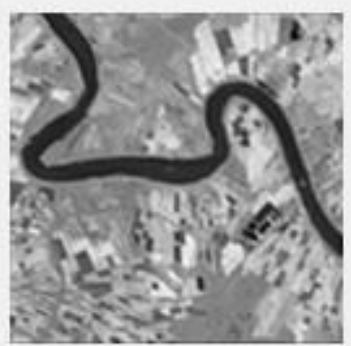




\section{International Journal of Innovative Research in Electrical, Electronics, Instrumentation and Control Engineering}

Vol. 6, Issue 12, December 2018

\begin{tabular}{|l|l|l|l|}
\hline S.no & Technique & MSE & PSNR \\
\hline 1 & DT-CWT NLM RE & 0.0174 & 17.5895 \\
\hline 2 & Proposed method & 0.186457 & 20.575035 \\
\hline
\end{tabular}

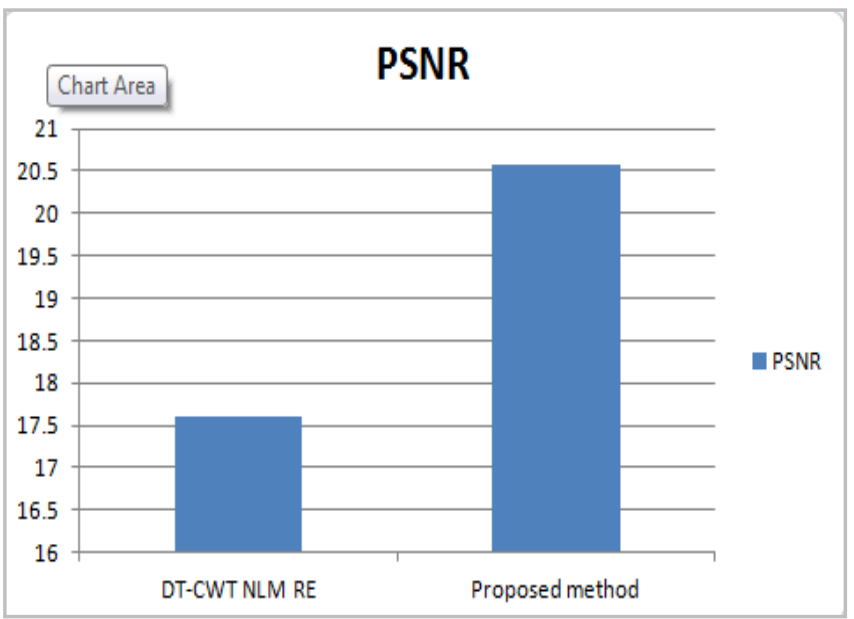

\section{CONCLUSION}

This paper provides the enhancement approach for the satellite image. This paper uses the method of double density dual tree complex wavelet transform and interpolation technique, based on that image enhancement is carried out. Edge preservation filtering named as non-local means filter (NLM) also used for suppressing undesirable information from high frequency details while interpolation happened. This system can be enhanced to detect changes between two images taken at different time for surveying the earth surface.

\section{REFERENCE}

[1] H. Demirel and G. Anbarjafari, "Image resolution enhancement by using discrete and stationary wavelet decomposition," IEEE Trans. Image Process., vol. 20, no. 5, pp. 1458-1460, May 2011

[2] H. Demirel and G. Anbarjafari, "Satellite image resolution enhancement using complex wavelet transform," IEEE Geosci Remote Sens. Lettvol. 7, no. 1, pp. 123-126, Jan. 2010.

[3] H. Demirel and G. Anbarjafari, "Image super resolution based on interpolation of wavelet domain high frequency subbands and the spatial domain input image,” ETRI J., vol. 32, no. 3, pp. 390-394, Jan. 2010.

[4] H. Zheng, A. Bouzerdoum, and S. L. Phung, "Wavelet based non-local means super-resolution for video sequences," in Proc. IEEE 17th Int. Conf. Image Process., Hong Kong, Sep. 26-29, 2010, pp. 2817-2820.

[5] A. Gambardella and M.Migliaccio, "On the super resolution of microwave scanning radiometer measurements," IEEE Geosci. Remote Sens. Lett., vol. 5, no. 4, pp. 796-800, Oct. 2008.

[6] A. Gambardella andM.Migliaccio, "On the superresolution of microwavescanning radiometer measurements," IEEE Geosci. Remote Sens. Lett.,vol. 5, no. 4, pp. 796-800, Oct. 2008.

[7] I. W. Selesnick, R. G. Baraniuk, and N. G. Kingsbur, “The dual-treecomplex wavelet transform,” IEEE Signal Prcess. Mag., vol. 22, no. 6,pp. 123-151, Nov. 2005

[8] J. L. Starck, F. Murtagh, and J. M. Fadili, Sparse Image and SignalProcessing: Wavelets, Curvelets, Morphological Diversity. Cambridge,U.K.: Cambridge Univ. Press, 2010.

[9] A. Buades, B. Coll, and J. M. Morel, “A review of image denoising algorithms, with a new one,” Multisc. Model.Simul., vol. 4, no. 2, pp. 490$530,2005$.

[10] J. Canny, “A computational approach to edge detection,” IEEE Trans. Pattern Anal. Mach. Intell., vol. PAMI-8, no. 6, pp. 679-698, Nov. 1986.

[11]Xinghao Ding, Xinxin Wang, Quan Xiao.”Color Image Enhancement with a Human Visual System Based Adaptive Filter,” 978-1-4244-55553/10/2010 IEEE

[12] M. N. Do and M. Vetterli, 2005, "The contourlet transform: An efficient directional multiresolution image representation," IEEE Trans. Image Proc., Vol.14, No. 12, pp. 2091-2106. 